\title{
Global Attractivity of a Periodic Delayed N-Species Model of Facultative Mutualism
}

\author{
Ahmadjan Muhammadhaji and Zhidong Teng \\ College of Mathematics and System Sciences, Xinjiang University, Urumqi 830046, China \\ Correspondence should be addressed to Zhidong Teng; zhidong@xju.edu.cn
}

Received 13 November 2012; Accepted 20 January 2013

Academic Editor: Beatrice Paternoster

Copyright (C) 2013 A. Muhammadhaji and Z. Teng. This is an open access article distributed under the Creative Commons Attribution License, which permits unrestricted use, distribution, and reproduction in any medium, provided the original work is properly cited.

Two classes of periodic $N$-species Lotka-Volterra facultative mutualism systems with distributed delays are discussed. Based on the continuation theorem of the coincidence degree theory developed by Gaines and Mawhin and the Lyapunov function method, some new sufficient conditions on the existence and global attractivity of positive periodic solutions are established.

\section{Introduction}

Mutualism is the interaction of two species of organisms that benefits both [1]. In general, mutualism may be either obligate or facultative. Obligate mutualist may survive only by association, and facultative mutualist, while benefiting from the presence of each other, may also survive in the absence of any of them [2]. As it is well known, in recent years the nonautonomous and periodic population dynamical systems are extensively studied. The basic and important studied questions for these systems are the persistence, permanence, and extinction of species, global stability of systems and the existence of positive periodic solutions, positive almost periodic solutions and strictly positive solutions, and so forth. Many important and influential results have been established and can be found in many articles and books. Particularly, the existence of positive periodic solutions for various type population dynamical systems has been extensively studied in [1-16] and the references cited therein.

In [7], the authors studied the following delayed twospecies model of facultative mutualism:

$$
\begin{aligned}
\dot{y}_{1}(t)=y_{1}(t) & {\left[r_{1}(t)-a_{1}(t) y_{1}(t)-b_{1}(t) y_{1}\left(t-\tau_{1}(t)\right)\right.} \\
+ & \left.c_{1}(t) y_{2}\left(t-p_{1}(t)\right)\right]
\end{aligned}
$$

$$
\begin{aligned}
\dot{y}_{2}(t)=y_{2}(t) & {\left[r_{2}(t)-a_{2}(t) y_{2}(t)-b_{2}(t) y_{2}\left(t-\tau_{1}(t)\right)\right.} \\
& \left.+c_{2}(t) y_{1}\left(t-p_{1}(t)\right)\right] .
\end{aligned}
$$

By using the technique of coincidence degree and the Lyapunov functionals method, the sufficient conditions for the existence and globally asymptotic stability of positive periodic solutions are obtained for system (1). In [2], the authors considered the following periodic delayed two-species model of facultative mutualism:

$$
\begin{aligned}
\dot{y}_{1}(t)=y_{1}(t) & {\left[r_{1}(t)-a_{1}(t) y_{1}(t)+b_{1}(t) y_{1}\left(t-\tau_{1}(t)\right)\right.} \\
& \left.+c_{1}(t) y_{2}\left(t-p_{1}(t)\right)\right], \\
\dot{y}_{2}(t)=y_{2}(t) & {\left[r_{2}(t)-a_{2}(t) y_{2}(t)+b_{2}(t) y_{2}\left(t-\tau_{1}(t)\right)\right.} \\
& \left.+c_{2}(t) y_{1}\left(t-p_{1}(t)\right)\right] .
\end{aligned}
$$

By means of the methods of coincidence degree and the Lyapunov functional, the sufficient conditions for the existence and globally asymptotic stability of positive periodic solutions are established for system (2). In [12], the following $n$-species periodic Lotka-Volterra type competitive systems 
with feedback controls and finite and infinite distributed delays are discussed:

$$
\begin{gathered}
\dot{y}_{i}(t)=y_{i}(t)\left[r_{i}(t)-a_{i i}(t) y_{i}(t)\right. \\
\quad-\sum_{j=1, j \neq i}^{n} a_{i j}(t) \int_{0}^{\omega} K_{i j}(s) y_{j}(t-s) d s \\
\left.\quad-\alpha_{i}(t) \int_{0}^{\omega} H_{i}(s) u_{i}(t-s) d s\right], \\
\dot{u}_{i}(t)=-\eta_{i}(t)+a_{i}(t) \int_{0}^{\omega} K_{i}(s) y_{i}(t-s) d s,
\end{gathered}
$$

where $i=1,2, \ldots, n$. By using the technique of coincidence degree and the Lyapunov functionals method, the sufficient conditions for the existence and global stability of positive periodic solutions are obtained for system (3).

Motivated by the above works, in this paper, we investigate the following two classes of $n$ species periodic model of facultative mutualism with finite distributed delays:

$$
\begin{aligned}
\dot{x}_{i}(t)=x_{i}(t)[ & r_{i}(t)-\alpha_{i}(t) x_{i}(t) \\
& \left.+\sum_{j=1}^{n} \sum_{l=1}^{m} a_{i j l}(t) \int_{-\tau}^{0} k_{i j l}(s) x_{j}(t+s) d s\right] \\
\dot{x}_{i}(t)=x_{i}(t)\left[\begin{array}{l}
r_{i}(t)-\alpha_{i}(t) x_{i}(t) \\
\end{array}\right. & -\sum_{l=1}^{m} a_{i i l}(t) \int_{-\tau}^{0} k_{i i l}(s) x_{i}(t+s) d s \\
& \left.+\sum_{j \neq i}^{n} \sum_{l=1}^{m} a_{i j l}(t) \int_{-\tau}^{0} k_{i j l}(s) x_{j}(t+s) d s\right]
\end{aligned}
$$

By using the technique of coincidence degree developed by Gaines and Mawhin in [17] and the Lyapunov functional method, we will establish some new sufficient conditions which guarantee that the system has at least one positive periodic solution and is globally attractive.

The organization of this paper is as follows. In the next section we will present some basic assumptions and main definitions and lemmas. In Section 3, conditions for the existence and global attractivity of positive periodic solution. In Section 4, two examples are given to illustrate that our main results are applicable. In the final section, we will discuss what we study in this paper and what we had in this paper.

\section{Preliminaries}

In systems (4) and (5), we have that $x_{i}(t)(i=1,2, \ldots n)$ represent the density of $n$ species $x_{i}(i=1,2, \ldots n)$ at time $t$, respectively; $r_{i}(t)(i=1,2, \ldots n)$ represent the intrinsic growth rate of species $x_{i}(i=1,2, \ldots n)$ at time $t$, respectively; $\alpha_{i}(t)(i=1,2, \ldots, n)$ represent the intrapatch restriction density of species $x_{i}(i=1,2, \ldots n)$ at time $t$, respectively; $a_{i j l}(t)(l=1,2, \ldots, m, i \neq j, i, j=1,2, \ldots, n)$ represent the mutualism coefficients between $n$ species $x_{i}(i=1,2, \ldots n)$ at time $t$, respectively, while $\tau \geq 0$ is a constant and $\tau$ may be $+\infty$. System (4) involves positive feedback terms

$$
\sum_{l=1}^{m} a_{i i l}(t) \int_{-\tau}^{0} k_{i i l}(s) x_{i}(t+s) d s, \quad i=1,2, \ldots, n
$$

which can be regarded as the passive effect on the growth rate of a species, and system (5) involves negative feedback terms

$$
-\sum_{l=1}^{m} a_{i i l}(t) \int_{-\tau}^{0} k_{i i l}(s) x_{i}(t+s) d s, \quad i=1,2, \ldots, n
$$

which are due to gestation. In this paper, we always assume the following:

(H1) $r_{i}(t)$ are $\omega$-periodic continuous functions with $\int_{0}^{\omega} r_{i}(t) d t>0(i=1,2, \ldots, n) ; \alpha_{i}(t)(i=1,2, \ldots, n)$ and $a_{i j l}(t)(i, j=1,2, \ldots, n ; l=1,2, \ldots, m)$ are positive $\omega$-periodic continuous functions; $k_{i j l}(s)(i, j=1,2, \ldots, n ; l=1,2, \ldots, m)$ are nonnegative integrable functions on $[-\tau, 0]$ satisfying $\int_{-\tau}^{0} k_{i j l}(s) d s=1$.

From the viewpoint of mathematical biology, in this paper for systems (4) and (5) we only consider the solution with the following initial condition:

$$
x_{i}(t)=\phi_{i}(s), \quad \forall s \in[-\tau, 0], i=1,2, \ldots, n,
$$

where $\phi_{i}(s)(i=1,2, \ldots, n)$ are nonnegative continuous functions defined on $[-\tau, 0]$ satisfying $\phi_{i}(0)>0(i=1,2, \ldots, n)$.

In this paper, for any $\omega$-periodic continuous function $f(t)$ we denote the following:

$$
\begin{gathered}
f^{L}=\min _{t \in[0, \omega]} f(t), \quad f^{M}=\max _{t \in[0, \omega]} f(t), \\
\bar{f}=\frac{1}{\omega} \int_{0}^{\omega} f(t) d t .
\end{gathered}
$$

In order to obtain the existence of positive $\omega$-periodic solutions of systems (4) and (5), we will use the continuation theorem developed by Gaines and Mawhin in [17]. For the reader's convenience, we will introduce the continuation theorem in the following.

Let $X$ and $Z$ be two normed vetor spaces. Let $L: \operatorname{Dom} L \subset$ $X \rightarrow Z$ be a linear operator and $N: X \rightarrow Z$ be a continuous operator. The operator $L$ is called a Fredholm operator of index zero, if $\operatorname{dim} \operatorname{Ker} L=\operatorname{codim} \operatorname{Im} L<\infty$ and $\operatorname{Im} L$ is a closed set in $Z$. If $L$ is a Fredholm operator of index 
zero, then there exist continuous projectors $P: X \rightarrow X$ and $Q: Z \rightarrow Z$ such that $\operatorname{Im} P=\operatorname{Ker} L$ and $\operatorname{Im} L=$ $\operatorname{Ker} Q=\operatorname{Im}(I-Q)$. It follows that $L \mid \operatorname{Dom} L \cap \operatorname{Ker} P$ : Dom $L \cap \operatorname{Ker} P \rightarrow \operatorname{Im} L$ is invertible and its inverse is denoted by $K_{P}$ and denote by $J: \operatorname{Im} Q \rightarrow$ Ker $L$ an isomorphism of $\operatorname{Im} Q$ onto $\operatorname{Ker} L$. Let $\Omega$ be a bounded open subset of $X$, we say that the operator $N$ is $L$-compact on $\bar{\Omega}$, where $\bar{\Omega}$ denotes the closure of $\Omega$ in $X$, if $Q N(\bar{\Omega})$ is bounded and $K_{P}(I-Q) N$ : $\bar{\Omega} \rightarrow X$ is compact.

Lemma 1 (see [17]). Let $L$ be a Fredholm operator of index zero and let $N$ be L-compact on $\bar{\Omega}$. If

(a) for each $\lambda \in(0,1)$ and $x \in \partial \Omega \cap \operatorname{Dom} L, L x \neq \lambda N x$;

(b) for each $x \in \partial \Omega \cap \operatorname{Ker} L, Q N x \neq 0$;

(c) $\operatorname{deg}\{J Q N, \Omega \cap \operatorname{Ker} L, 0\} \neq 0$,

then the operator equation $L x=N x$ has at least one solution lying in $\operatorname{Dom} L \cap \bar{\Omega}$.

\section{Main Results}

Now, for the convenience of statements, we denote the function

$$
a_{i j}(t)=\sum_{l=1}^{m} a_{i j l}(t), \quad i, j=1,2, \ldots, n .
$$

The following theorem is about the existence and global attractivity of positive periodic solutions of system (4).

Theorem 2. Suppose that assumption (H1) holds and there exists a constant $\mu_{i}>0(i=1,2, \ldots, n)$ such that

$$
\begin{gathered}
\min _{t \in[0, \omega]}\left\{\mu_{i} \alpha_{i}(t)-\sum_{j=1}^{n} \sum_{l=1}^{m} \mu_{j} \int_{-\tau}^{0} a_{j i l}(t-s) k_{j i l}(s) d s\right\} \\
=: \delta_{i}>0, \quad i=1,2, \ldots, n,
\end{gathered}
$$

and the algebraic equation

$$
\bar{r}_{i}-\bar{\alpha}_{i} v_{i}+\sum_{j=1}^{n} \bar{a}_{i j} v_{j}=0, \quad i=1,2, \ldots, n
$$

has a unique positive solution. Then, system (4) has a positive $\omega$-periodic solution which is globally attractive.

Proof. We firstly consider the existence of positive periodic solutions of system (4). For system (4), we introduce new variables $u_{i}(t)(i=1,2, \ldots, n)$ such that

$$
x_{i}(t)=\exp \left\{u_{i}(t)\right\}, \quad i=1,2, \ldots, n .
$$

Then, system (4) is rewritten in the following form:

$$
\begin{array}{r}
\dot{u}_{i}(t)=r_{i}(t)-\alpha_{i}(t) \exp \left\{u_{i}(t)\right\} \\
+\sum_{l=1}^{m} a_{i i l}(t) \int_{-\tau}^{0} k_{i i l}(s) \exp \left\{u_{i}(t+s)\right\} d s \\
+\sum_{j \neq i}^{n} \sum_{l=1}^{m} a_{i j l}(t) \int_{-\tau}^{0} k_{i j l}(s) \exp \left\{u_{j}(t+s)\right\} d s, \\
i=1,2, \ldots, n .
\end{array}
$$

In order to apply Lemma 1 to system (14), we introduce the normed vector spaces $X$ and $Z$ as follows. Let $C\left(R, R^{n}\right)$ denote the space of all continuous function $u(t)=$ $\left(u_{1}(t), u_{2}(t), \ldots, u_{n}(t)\right): R \rightarrow R^{n}$. We take

$$
X=Z=\left\{u(t) \in C\left(R, R^{n}\right): u(t)\right.
$$

is an $\omega$-periodic function $\}$

with norm

$$
\|u\|=\sum_{i=1}^{n} \max _{t \in[0, \omega]}\left|u_{i}(t)\right| .
$$

It is obvious that $X$ and $Z$ are the Banach spaces.

We define a linear operator $L:$ Dom $L \subset X \rightarrow Z$ and a continuous operator $N: X \rightarrow Z$ as follows:

$$
\begin{gathered}
L u(t)=\dot{u}(t), \\
N u(t)=\left(N u_{1}(t), N u_{2}(t), \ldots, N u_{n}(t)\right),
\end{gathered}
$$

where

$$
\begin{array}{r}
N u_{i}(t)=r_{i}(t)-\alpha_{i}(t) \exp \left\{u_{i}(t)\right\} \\
+\sum_{l=1}^{m} a_{i i l}(t) \int_{-\tau}^{0} k_{i i l}(s) \exp \left\{u_{i}(t+s)\right\} d s \\
+\sum_{j \neq i}^{n} \sum_{l=1}^{m} a_{i j l}(t) \int_{-\tau}^{0} k_{i j l}(s) \exp \left\{u_{j}(t+s)\right\} d s, \\
i=1,2, \ldots, n .
\end{array}
$$

Further, we define continuous projectors $P: X \rightarrow X$ and $Q: Z \rightarrow Z$ as follows:

$$
P u(t)=\frac{1}{\omega} \int_{0}^{\omega} u(t) d t, \quad Q v(t)=\frac{1}{\omega} \int_{0}^{\omega} v(t) d t .
$$

We easily see that $\operatorname{Im} L=\left\{v \in Z: \int_{0}^{\omega} v(t) d t=0\right\}$ and $\operatorname{Ker} L=$ $R^{n}$. It is obvious that $\operatorname{Im} L$ is closed in $Z$ and $\operatorname{dim} \operatorname{Ker} L=n$. Since for any $v \in Z$ there are unique $v_{1} \in R^{n}$ and $v_{2} \in \operatorname{Im} L$ with

$$
v_{1}=\frac{1}{\omega} \int_{0}^{\omega} v(t) d t, \quad v_{2}(t)=v(t)-v_{1}
$$

such that $v(t)=v_{1}+v_{2}(t)$, we have co $\operatorname{dim} \operatorname{Im} L=n$. Therefore, $L$ is a Fredholm mapping of index zero. Furthermore, the 
generalized inverse (to $L$ ) $K_{p}: \operatorname{Im} L \rightarrow \operatorname{Ker} P \cap \operatorname{Dom} L$ is given in the following form:

$$
K_{p} v(t)=\int_{0}^{t} v(s) d s-\frac{1}{\omega} \int_{0}^{\omega} \int_{0}^{t} v(s) d s d t .
$$

For convenience, we denote $F(t)=\left(F_{1}(t), F_{2}(t), \ldots, F_{n}(t)\right)$ as follows:

$$
\begin{array}{r}
F_{i}(t)=r_{i}(t)-\alpha_{i}(t) \exp \left\{u_{i}(t)\right\} \\
+\sum_{l=1}^{m} a_{i i l}(t) \int_{-\tau}^{0} k_{i i l}(s) \exp \left\{u_{i}(t+s)\right\} d s \\
+\sum_{j \neq i}^{n} \sum_{l=1}^{m} a_{i j l}(t) \int_{-\tau}^{0} k_{i j l}(s) \exp \left\{u_{j}(t+s)\right\} d s, \\
i=1,2, \ldots n .
\end{array}
$$

Thus, we have

$$
\begin{gathered}
Q N u(t)=\frac{1}{\omega} \int_{0}^{\omega} F(t) d t \\
K_{p}(I-Q) N u(t)=K_{p} I N u(t)-K_{p} Q N u(t) \\
=\int_{0}^{t} F(s) d s-\frac{1}{\omega} \int_{0}^{\omega} \int_{0}^{t} F(s) d s d t \\
+\left(\frac{1}{2}-\frac{t}{\omega}\right) \int_{0}^{\omega} F(s) d s .
\end{gathered}
$$

From formulas (23), we easily see that $Q N$ and $K_{p}(I-Q) N$ are continuous operators. Furthermore, it can be verified that $\overline{K_{p}(I-Q) N(\bar{\Omega})}$ is compact for any open bounded set $\Omega \subset$ $X$ by using Arzela-Ascoli theorem and $Q N(\bar{\Omega})$ is bounded. Therefore, $N$ is $L$-compact on $\bar{\Omega}$ for any open bounded subset $\Omega \subset X$.

Now, we reach the position to search for an appropriate open bounded subset $\Omega$ for the application of the continuation theorem (Lemma 1) to system (4).

Corresponding to the operator equation $L u(t)=\lambda N u(t)$ with parameter $\lambda \in(0,1)$, we have

$$
\dot{u}_{i}(t)=\lambda F_{i}(t), \quad i=1,2, \ldots, n,
$$

where $F_{i}(t)(i=1,2, \ldots, n)$ is given in (22).

Assume that $u(t)=\left(u_{1}(t), u_{2}(t), \ldots, u_{n}(t)\right) \in X$ is a solution of system (24) for some parameter $\lambda \in(0,1)$. By integrating system $(24)$ with the interval $[0, \omega]$, we obtain the following:

$$
\begin{aligned}
& \int_{0}^{\omega}\left[r_{i}(t)-\alpha_{i}(t) \exp \left\{u_{i}(t)\right\}\right. \\
& \quad+\sum_{l=1}^{m} a_{i i l}(t) \int_{-\tau}^{0} k_{i i l}(s) \exp \left\{u_{i}(t+s)\right\} d s \\
& \left.+\sum_{j \neq i}^{n} \sum_{l=1}^{m} a_{i j l}(t) \int_{-\tau}^{0} k_{i j l}(s) \exp \left\{u_{j}(t+s)\right\} d s\right] d t=0, \\
& i=1,2, \ldots n .
\end{aligned}
$$

Consequently,

$$
\begin{aligned}
\int_{0}^{\omega}\left[\alpha_{i}(t) \exp \left\{u_{i}(t)\right\}\right. \\
\quad-\sum_{l=1}^{m} a_{i i l}(t) \int_{-\tau}^{0} k_{i i l}(s) \exp \left\{u_{i}(t+s)\right\} d s \\
\left.\quad-\sum_{j \neq i}^{n} \sum_{l=1}^{m} a_{i j l}(t) \int_{-\tau}^{0} k_{i j l}(s) \exp \left\{u_{j}(t+s)\right\} d s\right] d t \\
=\bar{r}_{i} \omega, \quad i=1,2, \ldots n .
\end{aligned}
$$

From the continuity of $u(t)=\left(u_{1}(t), u_{2}(t), \ldots, u_{n}(t)\right)$, there exist constants $\xi_{i}, \eta_{i} \in[0, \omega](i=1,2, \ldots, n)$ such that

$$
\begin{aligned}
u_{i}\left(\xi_{i}\right)=\max _{t \in[0, \omega]} u_{i}(t), \quad u_{i}\left(\eta_{i}\right) & =\min _{t \in[0, \omega]} u_{i}(t), \\
i & =1,2, \ldots, n .
\end{aligned}
$$

From (26) and (27), we obtain

$$
\int_{0}^{\omega} \alpha_{i}(t) \exp \left\{u_{i}\left(\xi_{i}\right)\right\} d t \geq \bar{r}_{i} \omega, \quad i=1,2, \ldots, n
$$

Therefore, we further have

$$
u_{i}\left(\xi_{i}\right) \geq \ln \left(\frac{\bar{r}_{i}}{\bar{\alpha}_{i}}\right), \quad i=1,2, \ldots, n .
$$


For each $i, j=1,2, \ldots, n$ and $l=1,2, \ldots, m$, we have

$$
\begin{gathered}
+\cdots+\int_{0}^{\omega}\left[\left(\alpha_{n}(t)\right.\right. \\
\left.\quad-\sum_{l=1}^{m}\left(\int_{-\tau}^{0} a_{n n l}(t-s) k_{n n l}(s)\right) d s\right) \\
\times \exp \left\{u_{n}(t)\right\} \\
-\sum_{j \neq n}^{n} \sum_{l=1}^{m}\left(\int_{-\tau}^{0} a_{n j l}(t-s) k_{n j l}(s) d s\right) \\
\left.\quad \times \exp \left\{u_{j}(t)\right\}\right] d t \\
=\int_{0}^{\omega}\left[\alpha_{1}(t)-\sum_{l=1}^{m}\left(\int_{-\tau}^{0} a_{11 l}(t-s) k_{11 l}(s) d s\right.\right. \\
\left.\left.+\sum_{j \neq 1}^{n} \int_{-\tau}^{0} a_{j 1 l}(t-s) k_{j 1 l}(s) d s\right)\right]
\end{gathered}
$$$$
\int_{0}^{\omega} a_{i j l}(t) \int_{-\tau}^{0} k_{i j l}(s) \exp \left\{u_{j}(t+s)\right\} d s d t
$$$$
=\int_{-\tau}^{0} \int_{0}^{\omega} a_{i j l}(t) k_{i j l}(s) \exp \left\{u_{j}(t+s)\right\} d t d s
$$$$
=\int_{-\tau}^{0} \int_{s}^{s+\omega} a_{i j l}(v-s) k_{i j l}(s) \exp \left\{u_{j}(v)\right\} d v d s
$$$$
=\int_{-\tau}^{0} \int_{0}^{\omega} a_{i j l}(v-s) k_{i j l}(s) \exp \left\{u_{j}(v)\right\} d v d s
$$$$
=\int_{0}^{\omega} \int_{-\tau}^{0} a_{i j l}(v-s) k_{i j l}(s) \exp \left\{u_{j}(v)\right\} d s d v
$$$$
=\int_{0}^{\omega}\left(\int_{-\tau}^{0} a_{i j l}(t-s) k_{i j l}(s) d s\right) \exp \left\{u_{j}(t)\right\} d t .
$$

$\times \exp \left\{u_{1}(t)\right\} d t$

Hence, from (26) we further obtain

$$
\begin{aligned}
& \int_{0}^{\omega}\left[\left(\alpha_{i}(t)-\sum_{l=1}^{m}\left(\int_{-\tau}^{0} a_{i i l}(t-s) k_{i i l}(s) d s\right)\right) \exp \left\{u_{i}(t)\right\}\right. \\
& \left.\quad-\sum_{j \neq i}^{n} \sum_{l=1}^{m}\left(\int_{-\tau}^{0} a_{i j l}(t-s) k_{i j l}(s) d s\right) \exp \left\{u_{j}(t)\right\}\right] d t \\
& =\bar{r}_{i} \omega, \quad i=1,2, \ldots, n .
\end{aligned}
$$

$$
\begin{gathered}
+\int_{0}^{\omega}\left[\alpha_{2}(t)-\sum_{l=1}^{m}\left(\int_{-\tau}^{0} a_{22 l}(t-s) k_{22 l}(s) d s\right.\right. \\
+\sum_{j \neq 2}^{n} \int_{-\tau}^{0} a_{j 2 l}(t-s) \\
\left.\left.\times k_{j 2 l}(s) d s\right)\right]
\end{gathered}
$$

$\times \exp \left\{u_{2}(t)\right\} d t$

$$
+\cdots+\int_{0}^{\omega}\left[\alpha_{n}(t)\right.
$$

Consequently,

$$
\begin{gathered}
\int_{0}^{\omega}\left[\left(\alpha_{1}(t)-\sum_{l=1}^{m}\left(\int_{-\tau}^{0} a_{11 l}(t-s) k_{11 l}(s) d s\right)\right) \exp \left\{u_{1}(t)\right\}\right. \\
\left.-\sum_{j \neq 1}^{n} \sum_{l=1}^{m}\left(\int_{-\tau}^{0} a_{1 j l}(t-s) k_{1 j l}(s) d s\right) \exp \left\{u_{j}(t)\right\}\right] d t \\
+\int_{0}^{\omega}\left[\left(\alpha_{2}(t)-\sum_{l=1}^{m}\left(\int_{-\tau}^{0} a_{22 l}(t-s) k_{22 l}(s) d s\right)\right)\right. \\
\quad \times \exp \left\{u_{2}(t)\right\}-\sum_{j \neq 2}^{n} \sum_{l=1}^{m}\left(\int_{-\tau}^{0} a_{2 j l}(t-s) k_{2 j l}(s) d s\right) \\
\left.\quad \times \exp \left\{u_{j}(t)\right\}\right] d t
\end{gathered}
$$

$$
\begin{gathered}
-\sum_{l=1}^{m}\left(\int_{-\tau}^{0} a_{n n l}(t-s) k_{n n l}(s) d s\right. \\
+\sum_{j \neq n}^{n} \int_{-\tau}^{0} a_{j n l}(t-s) \\
\left.\left.\times k_{j n l}(s) d s\right)\right]
\end{gathered}
$$

$\times \exp \left\{u_{n}(t)\right\} d t$$$
=\int_{0}^{\omega}\left[\alpha_{1}(t)\right.
$$$$
-\sum_{l=1}^{m}\left(\int _ { - \tau } ^ { 0 } \left[a_{11 l}(t-s) k_{11 l}(s)+\sum_{j \neq 1}^{n} a_{j 1 l}(t-s)\right.\right.
$$$$
\left.\left.\left.\times k_{j 1 l}(s)\right] d s\right)\right]
$$ 
$\times \exp \left\{u_{1}(t)\right\} d t$

$+\int_{0}^{\omega}\left[\alpha_{2}(t)-\sum_{l=1}^{m}\left(\int_{-\tau}^{0}\left[a_{22 l}(t-s) k_{22 l}(s)\right.\right.\right.$

$$
\begin{aligned}
& +\sum_{j \neq 2}^{n} a_{j 2 l}(t-s) \\
& \left.\left.\left.\times k_{j 2 l}(s)\right] d s\right)\right]
\end{aligned}
$$

$\times \exp \left\{u_{2}(t)\right\} d t$

$$
\begin{aligned}
& +\cdots+\int_{0}^{\omega}\left[\alpha_{n}(t)\right. \\
& -\sum_{l=1}^{m}\left(\int _ { - \tau } ^ { 0 } \left[a_{n n l}(t-s) k_{n n l}(s)\right.\right. \\
& +\sum_{j \neq n}^{n} a_{j n l}(t-s) \\
& \left.\left.\left.\times k_{j n l}(s)\right] d s\right)\right] \\
& \times \exp \left\{u_{n}(t)\right\} d t=\sum_{i=1}^{n} \bar{r}_{i} \omega .
\end{aligned}
$$

From the assumptions of Theorem 2, we can obtain

$$
\begin{array}{r}
\int_{0}^{\omega}\left[\alpha_{i}(t)-\sum_{l=1}^{m}\left(\int _ { - \tau } ^ { 0 } \left[a_{i i l}(t-s) k_{i i l}(s)\right.\right.\right. \\
+\sum_{j \neq i}^{n} a_{j i l}(t-s) \\
\left.\left.\left.\times k_{j i l}(s)\right] d s\right)\right]
\end{array}
$$

$\times \exp \left\{u_{i}(t)\right\} d t$

$$
\leq \sum_{i=1}^{n} \bar{r}_{i} \omega, \quad i=1,2, \ldots, n
$$

Hence,

$$
\delta_{i} \int_{0}^{\omega} \exp \left\{u_{i}(t)\right\} d t \leq \sum_{i=1}^{n} \bar{r}_{i} \omega, \quad i=1,2, \ldots, n .
$$

Consequently,

$$
\int_{0}^{\omega} \exp \left\{u_{i}(t)\right\} d t \leq \frac{\sum_{i=1}^{n} \bar{r}_{i} \omega}{\delta_{i}}, \quad i=1,2, \ldots, n .
$$

From (35), we further obtain

$$
u_{i}\left(\eta_{i}\right) \leq \ln \left(\frac{\sum_{i=1}^{n} \bar{r}_{i}}{\delta_{i}}\right), \quad i=1,2, \ldots, n .
$$

On the other hand, directly from system (14) we have

$$
\begin{aligned}
& \int_{0}^{\omega}\left|\dot{u}_{i}(t)\right| d t \\
& \quad \leq \int_{0}^{\omega}\left[\left|r_{i}(t)\right|+\alpha_{i}(t) \exp \left\{u_{i}(t)\right\}\right.
\end{aligned}
$$$$
+\sum_{l=1}^{m} a_{i i l}(t) \int_{-\tau}^{0} k_{i i l}(s) \exp \left\{u_{i}(t+s)\right\} d s
$$$$
+\sum_{j \neq 1}^{n} \sum_{l=1}^{m} a_{i j l}(t) \int_{-\tau}^{0} k_{i j l}(s)
$$$$
\left.\times \exp \left\{u_{j}(t+s)\right\} d s\right] d t
$$

$$
=\int_{0}^{\omega}\left|r_{i}(t)\right| d t
$$

$+\int_{0}^{\omega}\left[\alpha_{i}(t)+\sum_{l=1}^{m} \int_{-\tau}^{0} a_{i i l}(t-s) k_{i i l}(s) d s\right]$

$\times \exp \left\{u_{i}(t)\right\} d t$

$+\int_{0}^{\omega}\left(\sum_{j \neq i}^{n} \sum_{l=1}^{m} \int_{-\tau}^{0} a_{i j l}(t-s) k_{i j l}(s) d s\right)$

$\times \exp \left\{u_{j}(t)\right\} d t$

$\leq \int_{0}^{\omega}\left|r_{i}(t)\right| d t+\int_{0}^{\omega} \alpha_{i}(t) \exp \left\{u_{i}(t)\right\} d t$

$+\sum_{l=1}^{m} a_{i i l}^{M} \int_{0}^{\omega} \exp \left\{u_{i}(t)\right\} d t$

$+\sum_{j \neq i}^{n} \sum_{l=1}^{m} a_{i j l}^{M} \int_{0}^{\omega} \exp \left\{u_{j}(t)\right\} d t$

$\leq \overline{\left|r_{i}\right|} \omega+\sum_{j=1}^{n} \sum_{l=1}^{m} a_{i j l}^{M} \frac{\sum_{i=1}^{n} \bar{r}_{i} \omega}{\delta_{i}}$

$+\alpha_{i}^{M} \frac{\sum_{i=1}^{n} \bar{r}_{i} \omega}{\delta_{i}}, \quad i=1,2, \ldots, n$. 
From (36) and (37), we have, for any $t \in[0, \omega]$,

$$
\begin{array}{r}
u_{i}(t) \leq u_{i}\left(\eta_{i}\right)+\int_{0}^{\omega}\left|\dot{u}_{i}(t)\right| d t \leq \ln \left(\frac{\sum_{i=1}^{n} \bar{r}_{i}}{\delta_{i}}\right)+\overline{\left|r_{i}\right|} \omega \\
+\sum_{j=1}^{n} \sum_{l=1}^{m} a_{i j l}^{M} \frac{\sum_{i=1}^{n} \bar{r}_{i} \omega}{\delta_{i}}+\alpha_{i}^{M} \frac{\sum_{i=1}^{n} \bar{r}_{i} \omega}{\delta_{i}}=: M_{i}, \\
i=1,2, \ldots, n .
\end{array}
$$

Further, from (29) and (37), we have, for any $t \in[0, \omega]$,

$$
\begin{aligned}
u_{i}(t) \geq & u_{i}\left(\xi_{i}\right)-\int_{0}^{\omega}\left|\dot{u}_{i}(t)\right| d t \\
\geq & \ln \left(\frac{\bar{r}_{i}}{\bar{\alpha}_{i}}\right)-\overline{\left|r_{i}\right|} \omega-\sum_{j=1}^{n} \sum_{l=1}^{m} a_{i j l}^{M} \frac{\sum_{i=1}^{n} \bar{r}_{i} \omega}{\delta_{i}} \\
& -\alpha_{i}^{M} \frac{\sum_{i=1}^{n} \bar{r}_{i} \omega}{\delta_{i}}=: N_{i}, \quad i=1,2, \ldots, n .
\end{aligned}
$$

Therefore, from (38) and (39) we have

$$
\begin{array}{r}
\max _{t \in[0, \omega]}\left|u_{i}(t)\right| \leq \max \left\{\left|M_{i}\right|,\left|N_{i}\right|\right\}=: B_{i}, \\
i=1,2, \ldots, n .
\end{array}
$$

It can be seen that the constants $B_{i}(i=1,2, \ldots, n)$ are independent of parameter $\lambda \in(0,1)$.

For any $u=\left(u_{1}, u_{2}, \ldots, u_{n}\right) \in R^{n}$, from (18) we can obtain

$$
\mathrm{QNu}=\left(\mathrm{QN} u_{1}, \mathrm{QNu_{2 }}, \ldots, \mathrm{QN} u_{n}\right),
$$

where

$$
\begin{array}{r}
\text { QNu }=\bar{r}_{i}-\left(\bar{\alpha}_{i}-\bar{a}_{i i}\right) \exp \left\{u_{i}\right\}+\sum_{j \neq i}^{n} \bar{a}_{i j} \exp \left\{u_{j}\right\}, \\
i=1,2, \ldots, n .
\end{array}
$$

We consider the following algebraic equation:

$$
\bar{r}_{i}-\left(\bar{\alpha}_{i}-\bar{a}_{i i}\right) v_{i}+\sum_{j \neq i}^{n} \bar{a}_{i j} v_{j}=0, \quad i=1,2, \ldots, n .
$$

From the assumption of Theorem 2, the equation has a unique positive solution $v^{*}=\left(v_{1}^{*}, v_{2}^{*}, \ldots, v_{n}^{*}\right)$. Hence, the equation $Q N u=0$ has a unique solution $u^{*}=$ $\left(u_{1}^{*}, u_{2}^{*}, \ldots, u_{n}^{*}\right)=\left(\ln v_{1}^{*}, \ln v_{2}^{*}, \ldots, \ln v_{n}^{*}\right) \in R^{n}$.

Choosing constant $B>0$ large enough such that $\left|u_{1}^{*}\right|+$ $\left|u_{2}^{*}\right|+\cdots+\left|u_{n}^{*}\right|<B$ and $B>B_{1}+B_{2}+\cdots+B_{n}$, we define a bounded open set $\Omega \subset X$ as follows:

$$
\Omega=\{u \in X:\|u\|<B\} .
$$

It is clear that $\Omega$ satisfies conditions (a) and (b) of Lemma 1. On the other hand, by direct calculating we can obtain

$$
\begin{gathered}
\operatorname{deg}\{J Q N, \Omega \cap \operatorname{Ker} L,(0,0, \ldots, 0)\} \\
=\operatorname{sgn}\left|\begin{array}{cccc}
f_{u_{1}}^{1} & f_{u_{2}}^{1} & \cdots & f_{u_{n}}^{1} \\
f_{u_{1}}^{2} & f_{u_{2}}^{2} & \cdots & f_{u_{n}}^{2} \\
\cdots & \cdots & \cdots & \cdots \\
f_{u_{1}}^{n} & f_{u_{2}}^{n} & \cdots & f_{u_{n}}^{n}
\end{array}\right|
\end{gathered}
$$

where

$$
\begin{gathered}
f_{u_{j}}^{i}=-\left(\bar{\alpha}_{i}-\bar{a}_{i j}\right) \exp \left\{u_{j}^{*}\right\}, \quad i=j, \\
f_{u_{j}}^{i}=\bar{a}_{i j} \exp \left\{u_{j}^{*}\right\}, \quad i \neq j, \quad i, j=1,2, \ldots, n .
\end{gathered}
$$

From the assumption of Theorem 2, we have

$$
\left|\begin{array}{cccc}
f_{u_{1}}^{1} & f_{u_{2}}^{1} & \cdots & f_{u_{n}}^{1} \\
f_{u_{1}}^{2} & f_{u_{2}}^{2} & \cdots & f_{u_{n}}^{2} \\
\cdots & \cdots & \cdots & \cdots \\
f_{u_{1}}^{n} & f_{u_{2}}^{n} & \cdots & f_{u_{n}}^{n}
\end{array}\right| \neq 0 .
$$

From this, we finally have

$$
\operatorname{deg}\{J Q N, \Omega \cap \operatorname{Ker} L,(0,0, \ldots, 0)\} \neq 0 .
$$

This shows that $\Omega$ satisfies condition (c) of Lemma 1 . Therefore, system (14) has a $\omega$-periodic solution $u^{*}(t)=$ $\left(u_{1}^{*}(t), u_{2}^{*}(t), \ldots, u_{n}^{*}(t)\right) \in \bar{\Omega}$. Further, from (13), system $(4)$ has a positive $\omega$-periodic solution $x^{*}(t)=\left(x_{1}^{*}(t), x_{2}^{*}(t), \ldots\right.$, $\left.x_{n}^{*}(t)\right)$.

Next, we will consider the global attractivity of positive periodic solutions $x^{*}(t)=\left(x_{1}^{*}(t), x_{2}^{*}(t), \ldots, x_{n}^{*}(t)\right)$ of system (4). Choose positive constants $m_{i}>0, M_{i}>0$ such that

$$
m_{i} \leq x_{i}^{*}(t) \leq M_{i}, \quad i=1,2, \ldots, n
$$

From the assumption of Theorem 2 , there exists constant $\beta>$ 0 such that for all $t \geq 0$ we have

$$
\delta_{i} \geq \beta>0, \quad i=1,2, \ldots, n
$$

Let $\left(x_{1}(t), x_{2}(t), \ldots, x_{n}(t)\right)$ be any solution of system (4), we define Lyapunov function as follows:

$$
\begin{aligned}
V_{i}(t)= & \mu_{i}\left|\ln x_{i}^{*}(t)-\ln x_{i}(t)\right| \\
& +\sum_{j=1}^{n} \sum_{l=1}^{m} \mu_{j} \int_{-\tau}^{0} k_{i j l}(s) \int_{t+s}^{t} a_{i j l}(\theta-s) \\
& \times\left|x_{j}^{*}(\theta)-x_{j}(\theta)\right| d \theta d s .
\end{aligned}
$$


Calculating the upper right derivation of $V_{i}(t)$ along system (4) for $i=1,2, \ldots, n$, we have

$$
\begin{aligned}
D^{+} V_{i}(t)= & \operatorname{sign}\left(x_{i}^{*}(t)-x_{i}(t)\right) \\
\times & {\left[-\mu_{i} \alpha_{i}(t)\left(x_{i}^{*}(t)-x_{i}(t)\right)\right.} \\
& +\sum_{j=1}^{n} \sum_{l=1}^{m} \mu_{j} a_{i j l}(t) \int_{-\tau}^{0} k_{i j l}(s) \\
& \left.\times\left(x_{j}^{*}(t+\theta)-x_{j}(t+\theta)\right) d s\right] \\
& +\sum_{j=1}^{n} \sum_{l=1}^{m} \mu_{j} \int_{-\tau}^{0} a_{i j l}(t-s) k_{i j l}(s) d s\left|x_{j}^{*}(t)-x_{j}(t)\right| \\
& -\sum_{j=1}^{n} \sum_{l=1}^{m} \mu_{j} a_{i j l}(t) \int_{-\tau}^{0} k_{i j l}(s) \\
& \times\left|x_{j}^{*}(t+\theta)-x_{j}(t+\theta)\right| d s \\
\leq & -\mu_{i} \alpha_{i}(t)\left|x_{i}^{*}(t)-x_{i}(t)\right| \\
& +\sum_{j=1}^{n} \sum_{l=1}^{m} \mu_{j} \int_{-\tau}^{0} a_{i j l}(t-s) k_{i j l}(s) d s\left|x_{j}^{*}(t)-x_{j}(t)\right| .
\end{aligned}
$$

Further, we define a Lyapunov function as follows:

$$
V(t)=\sum_{i=1}^{n} V_{i}(t)
$$

Calculating the upper right derivation of $V(t)$, from (52) we finally can obtain, for all $t \geq 0$,

$$
D^{+} V(t) \leq-\sum_{i=1}^{n} \delta_{i}\left|x_{i}^{*}(t)-x_{i}(t)\right|
$$

Integrating from 0 to $t$ on both sides of (54) and by (50) produces

$$
V(t)+\beta \int_{0}^{t}\left(\sum_{i=1}^{n}\left|x_{i}^{*}(s)-x_{i}(s)\right|\right) d s \leq V(0), \quad t \geq 0,
$$

then

$$
\int_{0}^{t}\left(\sum_{i=1}^{n}\left|x_{i}^{*}(s)-x_{i}(s)\right|\right) d s \leq \frac{V(0)}{\beta}, \quad t \geq 0 .
$$

By the definition of $V(t)$ and (53), we have

$$
\sum_{i=1}^{n} \mu_{i}\left|\ln x_{i}^{*}(t)-\ln x_{i}(t)\right| \leq V(t) \leq V(0), \quad t \geq 0 .
$$

Therefore, for $i=1,2, \ldots, n$ we have

$$
\mu_{i}\left|\ln x_{i}^{*}(t)-\ln x_{i}(t)\right| \leq V(0), \quad t \geq 0,
$$

which, together with (49), leads to

$$
\begin{aligned}
m_{i} \exp \left\{-\frac{V(0)}{\mu_{i}}\right\} & \leq x_{i}(t) \\
& \leq M_{i} \exp \left\{\frac{V(0)}{\mu_{i}}\right\}, \quad i=1,2, \ldots, n,
\end{aligned}
$$

and, hence, $\sum_{i=1}^{n}\left|x_{i}^{*}(t)-x_{i}(t)\right| \in L^{1}[0,+\infty)$. From the boundedness of $x_{i}^{*}(t)$ and (58), it follows that $x_{i}(t)(i=$ $1,2, \ldots, n)$ are bounded for $t \geq 0$. It is obvious that both $x_{i}(t)$ and $x_{i}^{*}(t)$ satisfy the equations of system (4), then by system (4) and the boundedness of $x_{i}(t)$ and $x_{i}^{*}(t)$, we know that the derivatives $\dot{x}_{i}(t)$ and $\dot{x}_{i}^{*}(t)$ are bounded. Furthermore, we can obtain that $\dot{x}_{i}^{*}(t)-\dot{x}_{i}(t)(i=1,2, \ldots, n)$ and their derivatives remain bounded on $[0,+\infty)$. Therefore $\sum_{i=1}^{n}\left|x_{i}^{*}(t)-x_{i}(t)\right|$ is uniformly continuous on $[0,+\infty)$. Thus, from (56), we have

$$
\lim _{t \rightarrow+\infty} \sum_{i=1}^{n}\left|x_{i}^{*}(t)-x_{i}(t)\right|=0
$$

Therefor,

$$
\lim _{t \rightarrow+\infty}\left(x_{i}^{*}(t)-x_{i}(t)\right)=0, \quad i=1,2, \ldots, n .
$$

This completes the proof of Theorem 2 .

From the proof of Theorem 2, on the existence and global attractivity of positive periodic solutions of system (5), we have the following result.

Corollary 3. Suppose that assumption (H1) holds and there exists a constant $\rho_{i}>0(i=1,2, \ldots, n)$ such that

$$
\begin{aligned}
& \min _{t \in[0, \omega]}\left\{\rho_{i} \alpha_{i}(t)-\sum_{j=1}^{n} \sum_{l=1}^{m} \rho_{j} \int_{-\tau}^{0} a_{j i l}(t-s) k_{j i l}(s) d s\right\} \\
& =: \lambda_{i}>0, \quad i=1,2, \ldots, n,
\end{aligned}
$$

and the algebraic equation

$$
\bar{r}_{i}-\left(\bar{\alpha}_{i}+\bar{a}_{i i}\right) v_{i}+\sum_{j \neq i}^{n} \bar{a}_{i j} v_{j}=0, \quad i=1,2, \ldots, n,
$$

has a unique positive solution. Then, system (5) has a positive $\omega$-periodic solution which is globally attractive. 


\section{Two Examples}

Example 4. First, we consider the following delayed system:

$$
\begin{aligned}
& \dot{x}_{1}(t)=x_{1}(t)\left[2+\cos (t)-(6+\cos (t)) x_{1}(t)\right. \\
& +\frac{2+\cos (t)}{3} \int_{-\tau}^{0} k_{111}(s) x_{1}(t+s) d s \\
& +\frac{2+\cos (t)}{3} \int_{-\tau}^{0} k_{121}(s) x_{2}(t+s) d s \\
& \left.+\frac{2+\cos (t)}{3} \int_{-\tau}^{0} k_{131}(s) x_{3}(t+s) d s\right] \text {, } \\
& \dot{x}_{2}(t)=x_{2}(t)\left[2+\cos (t)-(5+\cos (t)) x_{2}(t)\right. \\
& +\frac{2+\cos (t)}{7} \int_{-\tau}^{0} k_{221}(s) x_{2}(t+s) d s \\
& +\frac{3+\cos (t)}{4} \int_{-\tau}^{0} k_{211}(s) x_{1}(t+s) d s \\
& \left.+\frac{3+\cos (t)}{4} \int_{-\tau}^{0} k_{231}(s) x_{3}(t+s) d s\right], \\
& \dot{x}_{3}(t)=x_{3}(t)\left[2+\cos (t)-(6+\cos (t)) x_{3}(t)\right. \\
& +\frac{3+\cos (t)}{4} \int_{-\tau}^{0} k_{331}(s) x_{3}(t+s) d s \\
& +\frac{4+\cos (t)}{5} \int_{-\tau}^{0} k_{311}(s) x_{1}(t+s) d s \\
& \left.+\frac{3+\cos (t)}{5} \int_{-\tau}^{0} k_{321}(s) x_{2}(t+s) d s\right] \text {. }
\end{aligned}
$$

Corresponding to system (4), $n=3, m=1, \omega=2 \pi$, by direct calculation, we can get

$$
\sigma_{1} \approx 2, \quad \sigma_{2} \approx 1.6, \quad \sigma_{3} \approx 2.2
$$

and the following equations have unique positive solutions:

$$
\begin{aligned}
& \bar{a}_{11} v_{1}-\bar{a}_{12} v_{2}-\bar{a}_{13} v_{3}=\bar{r}_{1}, \\
& \bar{a}_{22} v_{1}-\bar{a}_{21} v_{2}-\bar{a}_{23} v_{3}=\bar{r}_{2}, \\
& \bar{a}_{33} v_{1}-\bar{a}_{31} v_{2}-\bar{a}_{32} v_{3}=\bar{r}_{3},
\end{aligned}
$$

where

$$
v_{1} \approx 0.1944, \quad v_{2} \approx 0.5625, \quad v_{3} \approx 0.8819 \text {. }
$$

It is clear that all the conditions of Theorem 2 hold. Hence, system (64) has a positive periodic solution which is globally attractive.
Example 5. Next, we consider the following delayed system:

$$
\begin{aligned}
\dot{x}_{1}(t)=x_{1}(t)[2 & +\cos (t)-\frac{5+4 \cos (t)}{20} x_{1}(t) \\
& +\frac{3+2 \cos (t)}{2} \int_{-\tau}^{0} k_{111}(s) x_{1}(t+s) d s \\
& +\frac{3+2 \cos (t)}{4} \int_{-\tau}^{0} k_{121}(s) x_{2}(t+s) d s \\
\dot{x}_{2}(t)=x_{2}(t)\left[2+\cos (t)-\frac{41+40 \cos (t)}{2} x_{-\tau}(t)\right. & \left.k_{131}(s) x_{3}(t+s) d s\right], \\
& +(2+\cos (t)) \int_{-\tau}^{0} k_{221}(s) x_{2}(t+s) d s \\
& +\frac{2+\cos (t)}{8} \int_{-\tau}^{0} k_{211}(s) x_{1}(t+s) d s \\
\dot{x}_{3}(t)=x_{3}(t)\left[2+\cos (t)-\frac{5+4 \cos (t)}{8} \int_{-\tau}^{0} k_{311}(s) x_{1}(t+s) d s\right. & \\
& \left.+\frac{3+2 \cos (t)}{4} \int_{-\tau}^{0} k_{231}(s) x_{3}(t+s) d s\right] \\
& +(4+\cos (t)) \int_{-\tau}^{0} k_{331}(s) x_{3}(t+s) d s \\
& \left.+\frac{5+3 \cos (t)}{8} \int_{321}^{0}(s) x_{2}(t+s) d s\right] .
\end{aligned}
$$

Corresponding to system (4), $n=3, m=1, \omega=2 \pi$, by direct calculation we can get

$$
\sigma_{1} \approx-5.2, \quad \sigma_{2} \approx-6.1, \quad \sigma_{3} \approx-7.2,
$$

and the following equations have a unique positive solutions:

$$
\begin{aligned}
& \bar{a}_{11} v_{1}-\bar{a}_{12} v_{2}-\bar{a}_{13} v_{3}=\bar{r}_{1}, \\
& \bar{a}_{22} v_{1}-\bar{a}_{21} v_{2}-\bar{a}_{23} v_{3}=\bar{r}_{2}, \\
& \bar{a}_{33} v_{1}-\bar{a}_{31} v_{2}-\bar{a}_{32} v_{3}=\bar{r}_{3},
\end{aligned}
$$

where

$$
v_{1}=-0.1622, \quad v_{2}=0.7007, \quad v_{3}=1.2717 .
$$

Clearly, the conditions of Theorem 2 do not hold.

From Figure 1 we can see that system (68) has no globally attractive positive periodic solution.

Remark 6. From these two examples, we can see that if the conditions of Theorem 2 hold, then the system has a globally attractive positive periodic solution. If the conditions of Theorem 2 do not hold, then the system has no globally attractive positive periodic solution. 

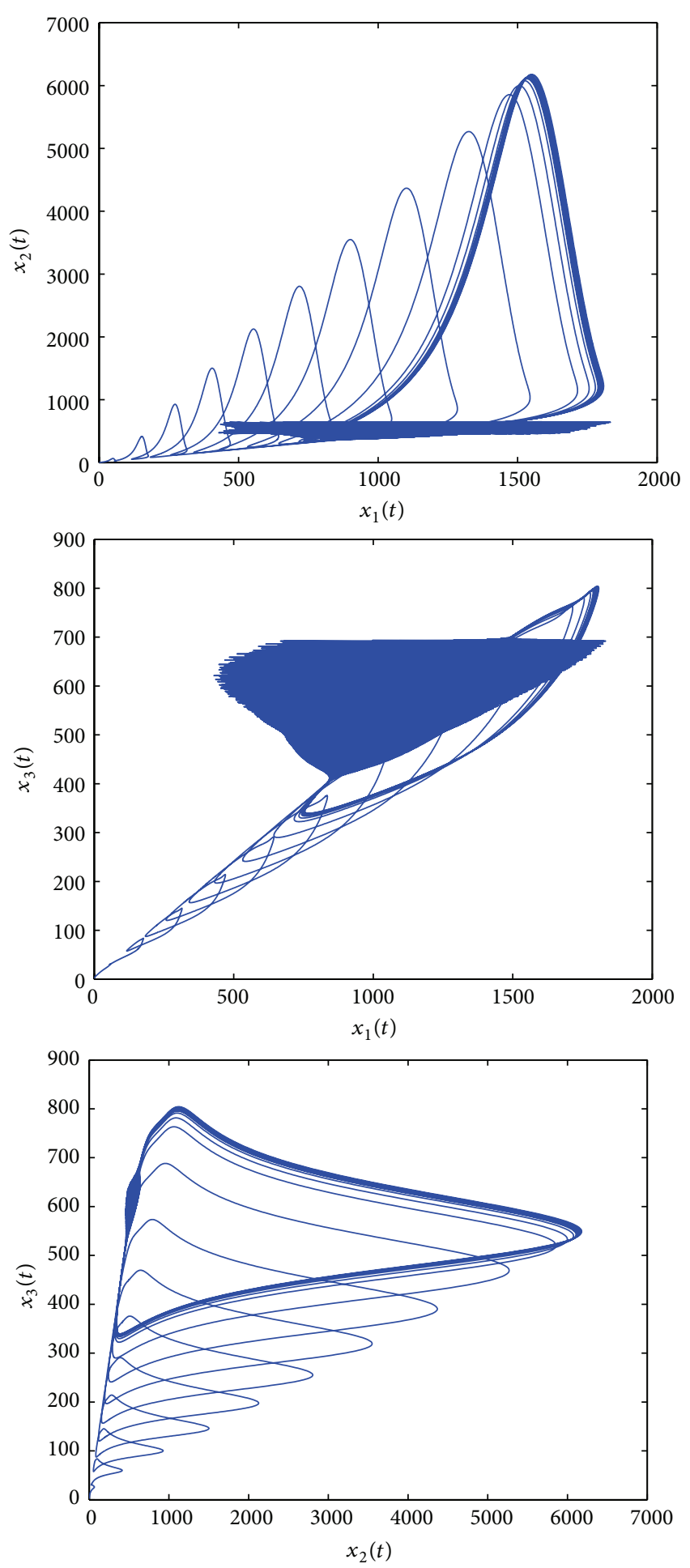

Figure 1: Numerical simulation for system (68). Here, we take the initial value $x_{0}=\left(x_{10}, x_{20}, x_{30}\right)=(1,1.5,2)$.

\section{Conclusions}

Mawhin's continuation theorem is a powerful tool for studying the existence of periodic solutions of periodic highdimensional time-delayed problems. When dealing with time-delayed problem, it is very convenient and the result is relatively simple. The most critical thing in the using of the theorem is the calculation of topological degree, that is, the condition (c) of the theorem.

In this paper, motivated by $[2,7]$ of Liu et al. we propose two classes of periodic $N$-species Lotka-Volterra facultative mutualism systems with distributed delays. By applying the continuation theorem of the coincidence degree theory developed by Gaines and Mawhin and the Lyapunov function method, we easily obtain sufficient conditions for the existence and global attractivity of positive periodic solutions of the system. From Theorem 2 and Corollary 3, we can see that the distributed time delays have effect on the existence and global attractivity of positive periodic solutions, and conditions (11) and (62) are very crucial to find the criteria for globally attractive positive periodic solutions. Further, the conditions (11) and (62) indicate that the undelayed intraspecific competition dominates the delayed intraspecific reproduction, and the intraspecific competition is more significant than the interspecific cooperation.

\section{Acknowledgments}

This work was supported by the National Natural Science Foundation of China (Grants nos. 11271312, 11261056, and 11261058), the China Postdoctoral Science Foundation (Grant no. 20110491750), and the Natural Science Foundation of Xinjiang (Grant no. 2011211B08).

\section{References}

[1] E. P. Odum, Fundamentals of Ecology, Saunders, Philadelphia, Pa, USA, 3rd edition, 1971.

[2] Z. Liu, J. Wu, R. Tan, and Y. Chen, "Modeling and analysis of a periodic delayed two-species model of facultative mutualism," Applied Mathematics and Computation, vol. 217, no. 2, pp. 893903, 2010.

[3] F. Chen, J. Shi, and X. Chen, "Periodicity in a Lotka-Volterra facultative mutualism system with several delays," Chinese Journal of Engineering Mathematics, vol. 21, no. 3, pp. 403-409, 2004.

[4] H. Wu, Y. Xia, and M. Lin, "Existence of positive periodic solution of mutualism system with several delays," Chaos, Solitons \& Fractals, vol. 36, no. 2, pp. 487-493, 2008.

[5] D. Hu and Z. Zhang, "Four positive periodic solutions to a Lotka-Volterra cooperative system with harvesting terms," Nonlinear Analysis: Real World Applications, vol. 11, no. 2, pp. 1115$1121,2010$.

[6] F. Hui and Z. Wang, "Existence and global attractivity of positive periodic solutions for delay Lotka-Volterra competition patch systems with stocking," Journal of Mathematical Analysis and Applications, vol. 293, no. 1, pp. 190-209, 2004.

[7] Z. Liu, R. Tan, Y. Chen, and L. Chen, "On the stable periodic solutions of a delayed two-species model of facultative mutualism," Applied Mathematics and Computation, vol. 196, no. 1, pp. 105-117, 2008.

[8] G. Lin and Y. Hong, "Periodic solutions to non autonomous predator prey system with delays," Nonlinear Analysis: Real World Applications, vol. 10, no. 3, pp. 1589-1600, 2009.

[9] S. Lu, "On the existence of positive periodic solutions to a Lotka Volterra cooperative population model with multiple delays," 
Nonlinear Analysis: Theory, Methods \& Applications, vol. 68, no. 6, pp. 1746-1753, 2008.

[10] J. Shen and J. Li, "Existence and global attractivity of positive periodic solutions for impulsive predator-prey model with dispersion and time delays," Nonlinear Analysis: Real World Applications, vol. 10, no. 1, pp. 227-243, 2009.

[11] F. Wei and K. Wang, "Global stability and asymptotically periodic solution for nonautonomous cooperative Lotka-Volterra diffusion system," Applied Mathematics and Computation, vol. 182, no. 1, pp. 161-165, 2006.

[12] P. Weng, "Existence and global stability of positive periodic solution of periodic integrodifferential systems with feedback controls," Computers \& Mathematics with Applications, vol. 40, no. 6-7, pp. 747-759, 2000.

[13] F. Yin and Y. Li, "Positive periodic solutions of a single species model with feedback regulation and distributed time delay," Applied Mathematics and Computation, vol. 153, no. 2, pp. 475484, 2004.

[14] C.-J. Zhao, L. Debnath, and K. Wang, "Positive periodic solutions of a delayed model in population," Applied Mathematics Letters, vol. 16, no. 4, pp. 561-565, 2003.

[15] L. Zhang, H.-X. Li, and X.-B. Zhang, "Periodic solutions of competition Lotka-Volterra dynamic system on time scales," Computers \& Mathematics with Applications, vol. 57, no. 7, pp. 1204-1211, 2009.

[16] Z. Zhang, J. Wu, and Z. Wang, "Periodic solutions of nonautonomous stagestructured cooperative system," Computers \& Mathematics With Applications, vol. 47, no. 4-5, pp. 699-706, 2004.

[17] R. E. Gaines and J. L. Mawhin, Coincidence Degree, and Nonlinear Differential Equations, Springer, Berlin, Germany, 1977. 


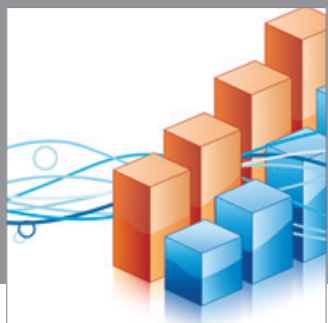

Advances in

Operations Research

mansans

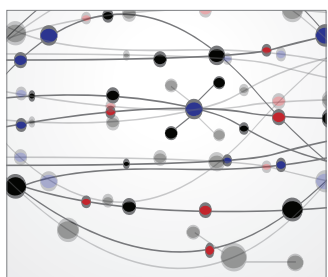

The Scientific World Journal
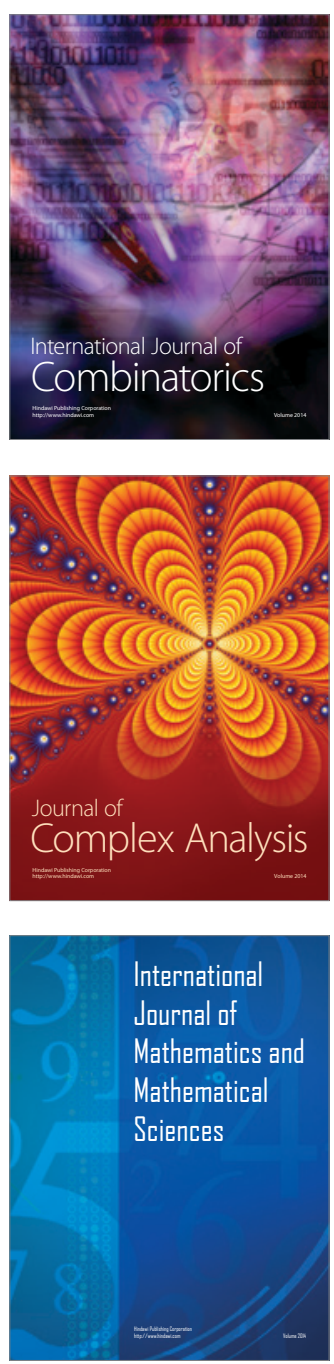
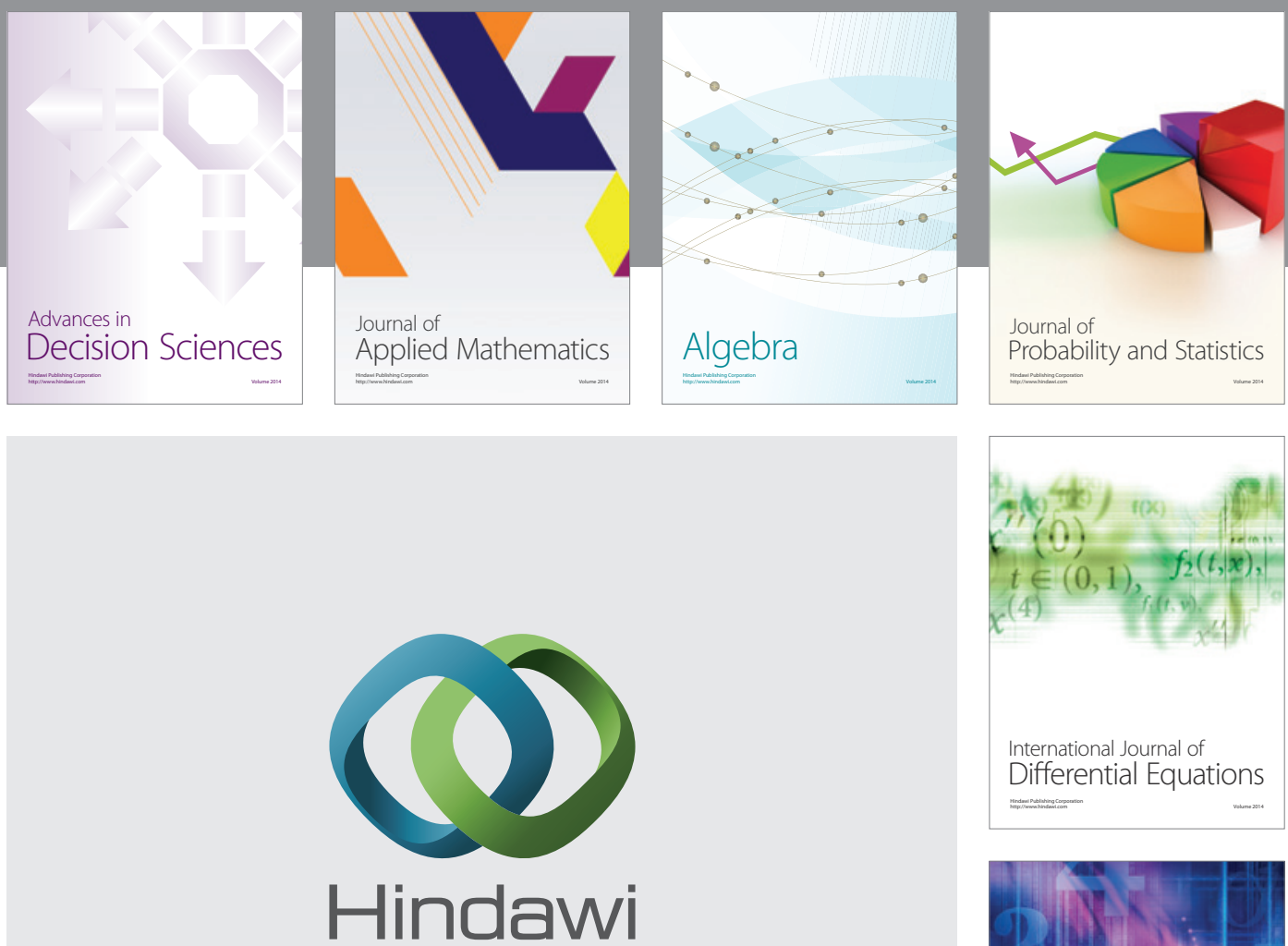

Submit your manuscripts at http://www.hindawi.com
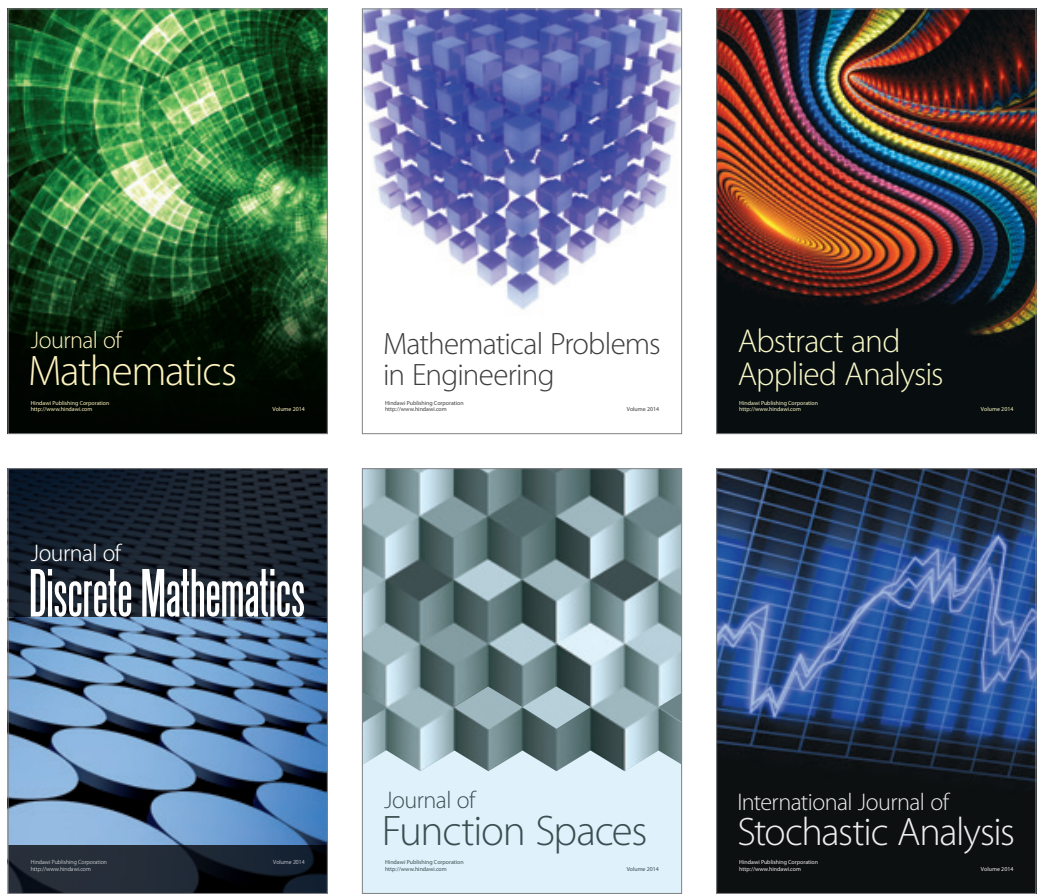

Journal of

Function Spaces

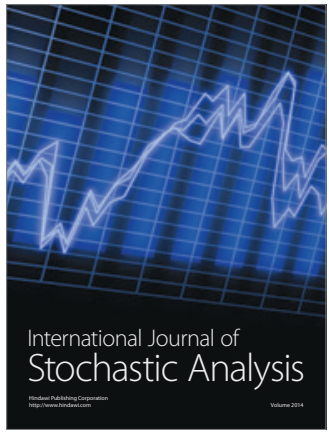

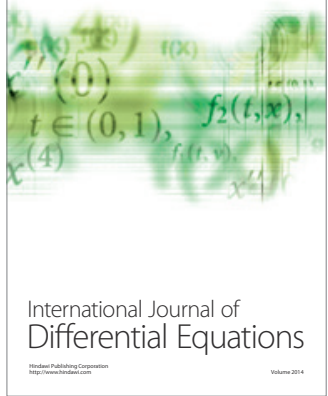
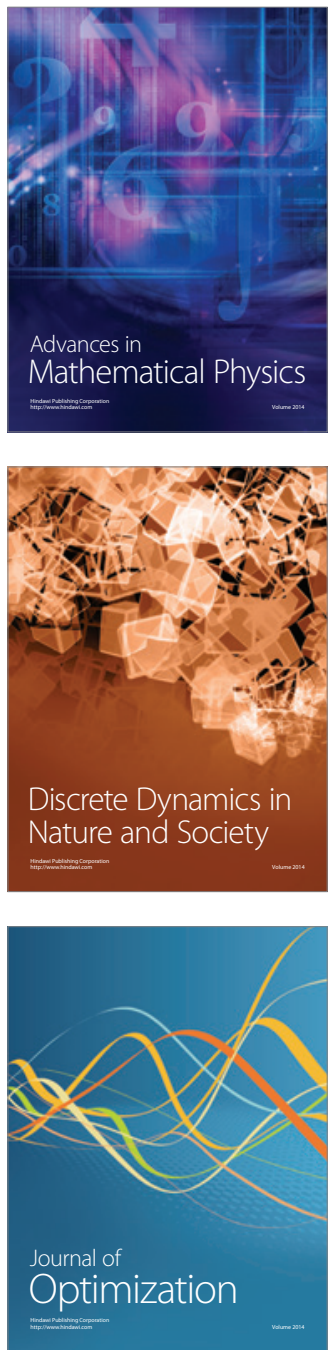